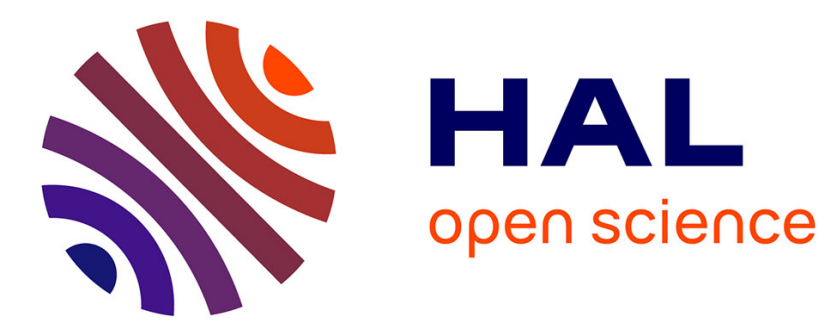

\title{
Physical Layer Split for User Selective Uplink Joint Reception in SDN Enabled Cloud-RAN
}

Dora Boviz, Aravinthan Gopalasingham, Chung Shue Chen, Laurent Roullet

\section{To cite this version:}

Dora Boviz, Aravinthan Gopalasingham, Chung Shue Chen, Laurent Roullet. Physical Layer Split for User Selective Uplink Joint Reception in SDN Enabled Cloud-RAN. AusCTW 2016 - 16th Australian Communications Theory Workshop , 2016, Melbourne, Australia. hal-01240998v2

\section{HAL Id: hal-01240998 \\ https://hal.inria.fr/hal-01240998v2}

Submitted on 11 Dec 2015

HAL is a multi-disciplinary open access archive for the deposit and dissemination of scientific research documents, whether they are published or not. The documents may come from teaching and research institutions in France or abroad, or from public or private research centers.
L'archive ouverte pluridisciplinaire HAL, est destinée au dépôt et à la diffusion de documents scientifiques de niveau recherche, publiés ou non, émanant des établissements d'enseignement et de recherche français ou étrangers, des laboratoires publics ou privés. 


\title{
Physical Layer Split for User Selective Uplink Joint Reception in SDN Enabled Cloud-RAN
}

\author{
Dora Boviz, Aravinthan Gopalasingham, Chung Shue Chen, Laurent Roullet \\ Alcatel-Lucent Bell Labs \\ Route de Villejust, 91620 Nozay, France \\ Emails: \{dora.boviz, gopalasingham.aravinthan, cs.chen, laurent.roullet\}@alcatel-lucent.com
}

\begin{abstract}
To meet quality of service requirements on the uplink of future cellular networks, we need to exploit intercell interference among users eligible for cooperation. Cloud Radio Access Network (C-RAN) architecture is particularly favorable to realize cooperation between users in neighboring cells, since signal detection is realized in the same processing unit. The novel technology of Software Defined Networking (SDN) increases the flexibility of network optimization and scalability of computational resources. We propose a C-RAN based architecture and a practical scheme of realizing uplink joint processing in critical scenarios where strong interference would affect cell-edge users. We consider characteristics of a real network and novel technological solutions necessary for reliable transmission over the radio access network. The central idea is to split the physical layer processing between Remote Radio Heads (RRHs) and the central processing unit only for selected users in enabling cooperation and maintaining affordable fronthaul transport infrastructure. In practice, the joint detection for selected few co-channel users would simplify the required multiuser channel estimation while improving overall performance and cell-edge users' quality-of-service (QoS).
\end{abstract}

\section{INTRODUCTION}

One of the main challenges for next generation cellular communication systems is to transmit with higher spectral efficiency, since frequency resources are limited but the amount of data traffic is growing very rapidly [1]. To be able to meet quality-of-service requirements, particularly for cell-edge users, without using large amount of bandwidth or transmit power, frequency reuse is applied. User equipments (UEs) transmitting on the same physical resource block (PRB) can lead to severe co-channel interference. Their transmissions can be accurately decoded if their respective base stations (BSs) cooperate [2]. Distributed and centralized schemes of cooperative processing were reported in the literature [3]. Decentralized processing was built for today's already deployed distributed RAN infrastructures. However, their cooperative processing gain remains quite limited. Its disadvantages such as high latency and large overhead needed for sharing data also orient the industry towards the use of a centralized architecture. In conventional uplink (UL) transmission, PRBs are reused for each cell. Inter-cell interference is expected and could lead to very low signal-to-interference-plus-noise ratio (SINR) especially to received signal from cell-edge user. Though the realization of large-scale multi-cell processing would be too complex, orchestration between the schedulers of each cell will allow better sharing of radio resources and joint detection (JD) of received signals. In this paper, we propose a centralized architecture in which users from neighboring cells can be allocated on the same PRB and their signals are detected jointly through sharing data between Baseband Units (BBUs) of their respective cells.

Exploiting inter-cell interference via multi-cell processing instead of avoidance [2] is one of the most interesting features for future cellular networks but also a very challenging task because of practical system limitations. Finite communication bandwidth between various network elements and potential multi-user channel estimation error [4] can easily decrease the system performance and expected theoretical gain. The trend is to use possible cooperation thanks to today's adoption of CRAN architecture. Here, we will discuss the use of software defined networking (SDN) technology for radio access networks, which offers the possibility of efficient network control by separating control plane and data plane. Low latency data flow routing using network-wide control information increases network scalability and adaptability for features such as joint detection.

The aim of this paper is to highlight UL inter-cell cooperation in a centralized framework. We first review and compare existing techniques in the light of their applicability to CRAN. We point out key innovations for allowing an efficient implementation of uplink coordinated multi-point transmission (CoMP). Then, we define an architecture with a physical layer (PHY) split that copes with system limitations and allows cooperation in today's C-RAN platform. Finally, we show by simulations and numerical studies the benefit of multicell cooperation which is implementable to today's Long Term Evolution (LTE) cellular networks.

We use the following technical terms in the coming discussion. The user or UE is the cellular device which sends data over the uplink channel. The data is then received by one or several antennas at the cell sites where only radio frequency or possibly physical layer signal processing is performed; we call them Remote Radio Heads (RRHs). If complete BBU is located there, it is then a base station (BS). Then the data is forwarded using fronthaul $(\mathrm{FH})$ links to the central processing unit hosting BBUs of several cells, which is called BBU-pool.

The remainder of the paper is organized as follows. In Section II, we describe the idea of uplink cooperation in cellular networks and existing techniques. Section III presents the proposed C-RAN architecture and implementation integrated with 
SDN controller and carrier Ethernet fronthauling. Section IV shows how these technological solutions enable the proposed user selective cooperation scheme and its performance. Finally, Section V concludes the paper.

\section{Uplink CoOperative PRocessing}

The idea of interference management through BS cooperation spans over several aspects of a cellular network regarding the amount of available channel state information (CSI) and shared data (compressed signal or decoded data), the $\mathrm{FH}$ network architecture (distributed or centralized processing), the communication infrastructures between BSs (e.g., fiber, microwave) and cooperation technique used (e.g., coordinated scheduling, joint detection). In this section, we investigate the state-of-the-art of uplink cooperative techniques already used in today's cellular networks or reported in standards and research papers.

\section{A. The Network MIMO concept}

In theory, we can assimilate multi-cell Multiple-Input Multiple-Output (MIMO) network without limitation in fronthaul connections to conventional multi-user MIMO (MUMIMO), so that in the uplink, with full cooperation, the capacity of each cell should be the same as that in an interferencefree scenario [5]. Unfortunately, this capacity result cannot be applied to real cellular networks due to several theoretical and practical constraints as already pointed out in [2]. Still, we can have several methods to exploit the inter-cell interference with fully or partially centralized signal detection [6]. Multi-cell joint detection can be realized like that in single-cell MIMO case using either Maximum Likelihood (ML) or Minimum Mean Square Error (MMSE) detection. However, the high complexity of ML does not allow implementation in real-time systems. On the other hand, joint MMSE is suboptimal but requires more reasonable computational cost. It is also possible to apply Successive Interference Cancellation (SIC) detection to each signal. The drawback of SIC is the possible error propagation if first signals cannot be accurately detected. A main difficulty of joint detection is to forward received signal to the collective processing unit (see sub-section II-C). Signal compression before sending on the fronthaul links can address this practical constraint, but can affect accuracy of detection.

Compression followed by SIC detection was studied in [7]. In [8] authors propose to forward to the BBU-pool a quantized version of demodulated symbols which is then used in joint processing. Amount of data sent over fronthaul links can also be reduced if a BS forwards already decoded signal that can be used to perform SIC for the other. Another form of decodeand-forward scheme is that a part of the message is individually decoded at the BS while the other part is forwarded for joint decoding in the BBU-pool [9]. Decentralized cooperation similar to decode-and-forward was investigated in [10], where decoded data is shared among neighboring BSs instead of a central unit. Where C-RAN architecture will be adopted in future cellular networks, we can benefit from low latency communication between collocated BBUs and consider more optimal scheme of multi-cell cooperation.

\section{B. UL CoMP in Long Term Evolution (LTE)}

In LTE, there are two classes of uplink CoMP: (i) coordinated scheduling (CS) which shares only control data between BBUs, and (ii) joint reception (JR) which requires large $\mathrm{FH}$ bandwidth to share received data signals. It should be noted that full multi-cell scheduling would also have a high computational complexity and cannot improve cell throughput beyond a limit in case of heavy load.

\section{Constraints: FH capacity and multi-cell channel estimation}

For reliable communication between RRHs and BBU-pool, low latency and low jitter links are required. Meanwhile, they have to be efficiently used for system cost. Optical fiber links of $10 \mathrm{Gbps}$ capacity transporting signals encapsulated in Common Public Radio Interface (CPRI) packets are often used as fronthaul links. However, in future networks that may coordinate many antennas at a cell site, this capacity would be a bottleneck to transmit I/Q samples. To offer a cost-effective solution, transmitting demodulated symbols or decoded bits between cell site and BBU-pool should be considered since this can significantly decrease the required $\mathrm{FH}$ bandwidth.

A basic constraint for CoMP is the necessity of multi-cell CSI at the central processing unit. Orthogonal pilot symbols need to be sent by each UE so that channel gain can be accurately estimated. If several users are transmitting on the same PRB, larger pilot-length is needed. This will consume extra bandwidth. Note that in LTE, cyclic shift of pilot symbols is proposed for multi-user signal detection [11]. However, the number of allowable co-channel users is still limited.

\section{Key TECHNOLOgIES FOR COOPERATIVE TRANSMISSIONS}

\section{A. $C-R A N$}

1) Advantages of centralization: As aforementioned, a centralized architecture can facilitate inter-cell cooperation. Where possible to deploy, C-RAN is particularly appropriate for low-latency communications between BBUs of different cells since these are located in the same processing unit. Centralization of BBUs also facilitates the handover procedure which otherwise would require coordination and communication between distributed BSs through for example the 3GPP $\mathrm{X} 2$ interface [12].

The need for such an architecture is not only for improving network performance, but also for reducing deployment and operational costs. To satisfy future data rate and coverage requirements, antenna density should be increased while operators' per-subscriber income decreases. In order to deploy denser networks at lower cost (the Capital Expenditure, CAPEX), processing capacity should be centralized so that system scalability, energy efficiency and flexibility to upgrade and manage computational units can be guaranteed, which will result in lower Operational Expenditure (OPEX) [13]. Since several BBUs are located in the same server, the overall 
dimension of computational resources can be reduced thanks to pooling. BBU-pools are also susceptible to be shared between several operators using the concept of RAN as a Service [14], which allows to have several instances of various standalone modules that can be attributed to different operators. Nonetheless, this centralization of BBUs requires resource management and data flow control. This can be provided by the emerging technology of SDN, to be discussed in subsection III-B.

2) Fronthaul transport using CPRI: In existing C-RAN deployments, RRHs are connected to BBU-pool through dedicated point-to-point fiber link on which synchronous CPRI packets containing baseband I/Q symbols are sent. As pointed out in [15], this type of infrastructure cannot meet future network requirements such as dynamic mapping and network resource allocation between RRHs and BBU-pool. Ethernet based Fronthaul technology connected through a CPRI-toEthernet gateway is a good candidate for transport CPRI packets [13]. Since today's FH cannot guarantee to transport baseband I/Q symbols for large scale MIMO transmissions, the common target of centralizing all the baseband processing cannot be ensured. In general, the distribution of PHY processing between the RRH and the BS depends on the available fronthaul rate measured on the BBU-pool side. However, to benefit from C-RAN and CoMP features, we have to design a dynamic architecture allowing the centralization of PHY functions.

\section{B. SDN controller in the BBU pool}

1) Data and control plane separation: The separation between control plane and data plane in SDN architecture is to enable them to operate independently for high flexibility and programmability so that operators can have a complete control over the network from a centralized point [16]. This would allow network operators to deploy their applications and services and to adjust network policies easily, see for example [17]. Load balancing and scheduling can also be easily deployed. Software defined networking has been used in wide variety of network environments such as enterprise networks, data centers and infrastructure-based wireless access networks.

2) OpenFlow for network management: OpenFlow has been the first protocol for SDN. It has two key components. The controller has a complete control over all the OpenFlow switches in the network, i.e., it controls all network functions, whereas the OpenFlow switch forwards the first packet of each new flow to the controller for routing decision. The controller can update the forwarding table depending on some pre-defined rules or new policies. Though OpenFlow has programmability and flexibility in managing and controlling various network elements in SDN, there are some concerns of its efficiency during heavy traffic load. DevoFlow [18] is a modification of OpenFlow protocol for maintaining flow visibility and reducing controller load and overhead. DevoFlow can reduce the switch flow table entries and also the control messages by more than ten times [18]. It would be very

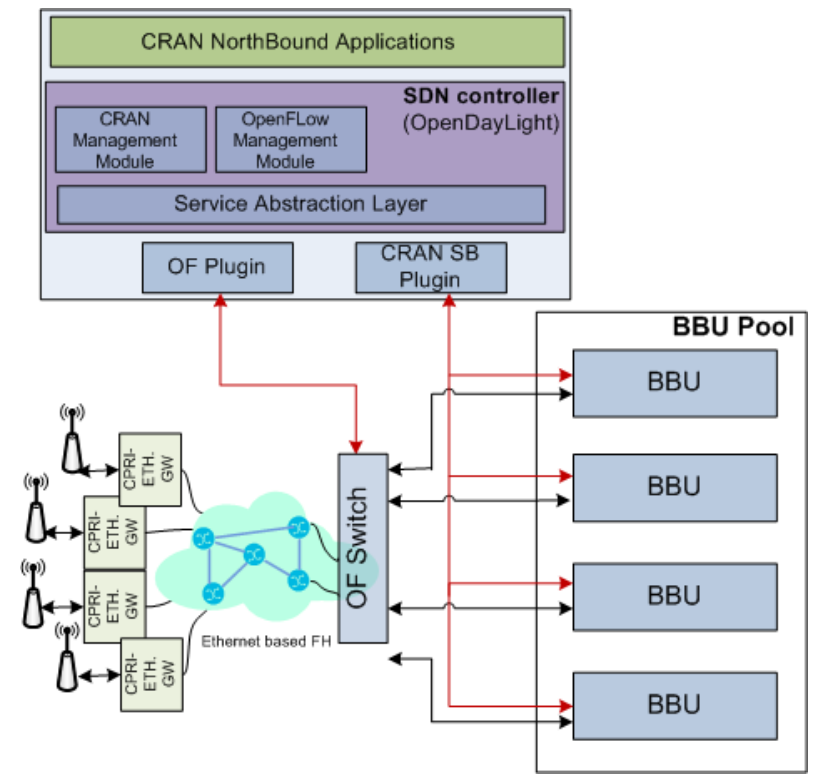

Fig. 1: C-RAN architecture with SDN controller.

suitable to offer efficient switching and routing solutions for high performance networks like C-RAN.

3) SDN approach for wireless networks: The importance of SDN is to bring dynamic programmability into the control and data planes. This will definitely also benefit mobile operators by incorporating SDN to wireless networks. OpenRoads [19] and OpenRadio [20] are examples of bringing SDN into RAN by enabling dynamic programmability of RAN especially at physical and MAC layer. In [21], generalized SDN framework for RAN is demonstrated based on OpenDayLight Controller for possible load balancing, interference management and dynamic radio resource sharing [13]. One can see that the flexibility, programmability and the single point control of SDN framework can support various objectives in C-RAN especially CoMP, where coordination between multiple BBUs is essential. It can also fit well for providing QoS-based dynamic switching between RRHs and BBUs in C-RAN and for sharing fronthaul network among operators.

Fig. 1 shows C-RAN with SDN controller. Signals are processed in the RRHs and the BBUs dedicated to each cell can communicate between one another inside the BBU-pool. The controller orchestrates data flows from RRHs towards the BBU-pool and inside it via control plane connections. Northbound (NB) applications are in charge of the coordination of specific features. Through the NB interface, they get measures and parameters from the controller and also send commands to the network and BBU-pool.

\section{Multicell Centralized Detection}

In the previous sections, we have studied C-RAN architecture with its key elements. This framework, by its centralized architecture, enables the execution of multicell joint MMSE detection function for selected users. A NB application of the SDN controller realizes the coordination of this feature by 


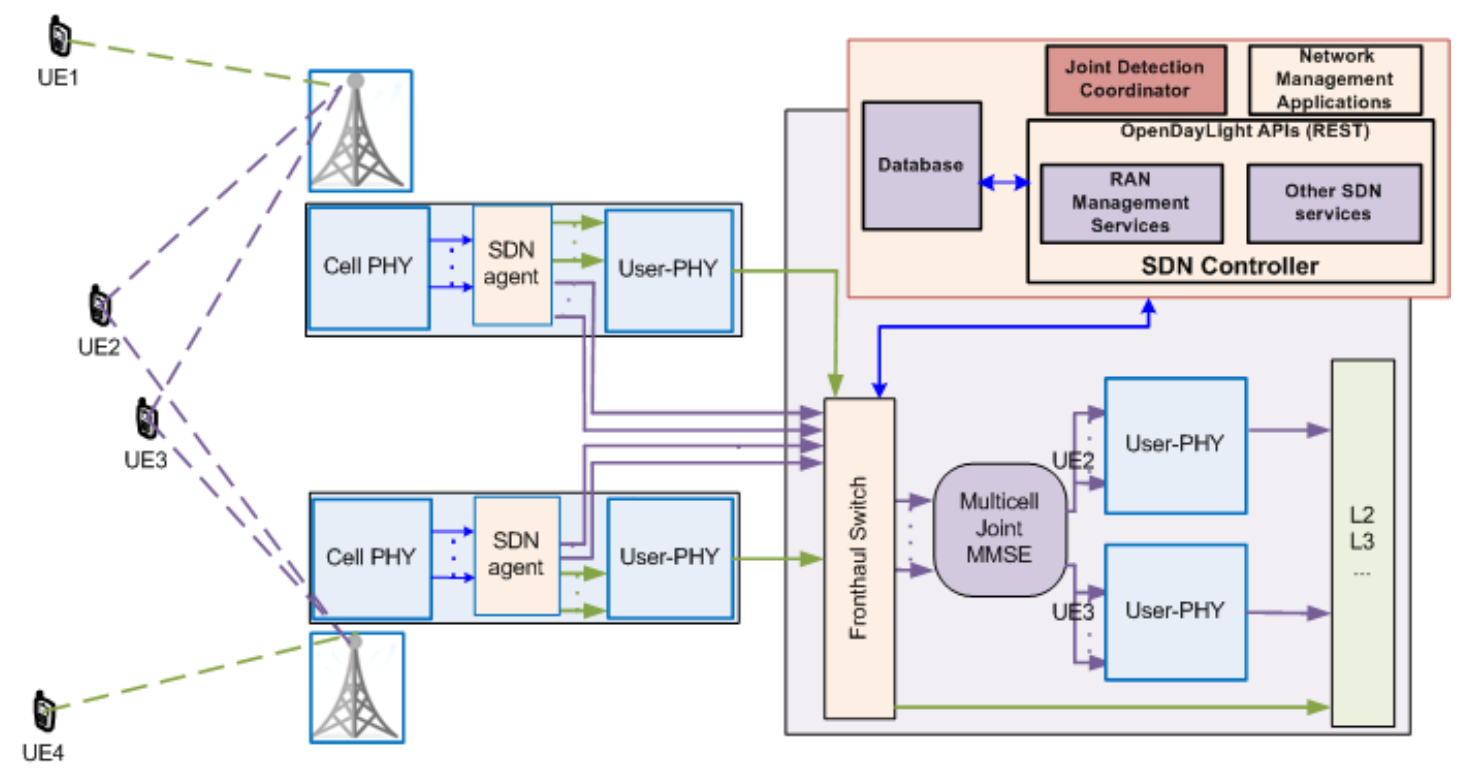

Fig. 2: Physical layer split for fronthaul enabling joint detection for selected users and user-PHY offload.

flow routing and signaling. We present the proposed physical layer split to enable joint processing and fronthaul offloading in Fig. 2, where Cell-PHY contains RF processing, cyclic prefix $(\mathrm{CP})$ removal, inverse fast Fourier transform (IFFT) and subcarrier demapping, and User-PHY includes inverse discrete Fourier transform (IDFT), demodulation, and parallel-to-serial conversion and decoding. Data flow routing is realized by the SDN agent in the RRH using information from the NB application on PRBs allocated to JD. For users not involved in the joint detection Cell- and User-PHY are both processed at the RRH, for other users I/Q symbols are forwarded to the BBU-pool to apply joint MMSE detection in order to retrieve QAM symbols sent by each UE.

\section{A. Use case}

In case of network congestion (heavy traffic load) in an area supported by several RRHs, severe interference for cell-edge users managed by Coordinated Scheduling would decrease their data rate. The more effective solution to provide reliable high data-rate uplink transmissions is to exploit interference signal from co-channel users through joint detection. We can schedule on the same PRB two interfering users and have their signals received by two RRHs. These received signals are then forwarded to the central processing unit, where we can use both of them to jointly detect the data sent by each user for high performance.

\section{B. Selective joint processing}

As expected, in large network, we cannot transmit every users' I/Q symbols to the BBU-pool even if high capacity fronthaul solution can be available. To realize efficient cooperation in C-RAN, we choose critical users who are suffering from high inter-cell interference and forward their I/Q samples to jointly process them in the BBU-pool with the signal of the interfering users. Other users' PHY processing is completed at the RRH and then signal is forwarded, so each one of them uses less than $5 \%$ of the fronthaul bandwidth needed for a JD user [22]. This would allow us to target cell-edge users in joint detection and hence improve overall performance.

As stated in [15], performing cell-related PHY processing at the BS would allow fronthaul capacity to be load-dependent. This feature is interesting and can be maintained by our design, since the PHY split for the joint detection is at the limit of cell-PHY and user-PHY processing. In addition, our approach makes a further decrease of required fronthaul capacity thanks to the possibility of performing user-PHY processing of nonselected users at the BS.

A difficulty for CoMP JD is joint multi-cell channel estimation at the receiver side. In LTE, cyclic shift is defined to enable multi-user channel estimation [23], i.e., Demodulation Reference Signals (DMRS) are shifted in order to make them orthogonal between users. Since we schedule only a limited number of users on the same PRB, we can use cyclic shift without being affected by the limitation of its signaling to 3 bits. Pilot symbols of users selected for joint detection need to be transferred as well to estimate their channel conditions to the receiver antennas, but again, for a few users it introduces less overhead than that required for large-scale joint detection.

\section{Coordination by a NB application of SDN controller}

An application connected to the SDN controller through its NB interface can activate joint detection and optimize user pairing and selection. Depending on applications, the architecture should allow any algorithm to be easily plugged for different optimization. Adaptability to various network dynamics is important. In our design, the SDN controller has access to all system measurements that are stored in the associated database. This allows network applications connected 
through the NB interface to ask for the measurements needed to run optimization algorithm. When the optimization is done, the controller sends instructions back to the network elements involved to execute.

For the cell coordination and joint detection, we propose the following process that aims at overall cell throughput improvement as well as good QoS for cell-edge users. To begin with, we consider cooperation in pairs of two cells and especially for cell-edge users, while cell-center users can transmit lower power for minimal interference. An adaptation of the proposed process can also be applied to joint detection in more than 2 cells.

- Step 1: Select users on the common edge of cooperating neighboring cells.

- Step 2: Pair these users, one from each cell in a pair (see in Fig. 2), by searching among these cell-edge users the sum-rate maximizing pair combination.

- Step 3: Select the user pair having the maximum sumrate.

- Step 4. If overall cell throughput of both cells increases by scheduling the selected user pair, activate joint detection for this pair. Iterate from Step 3.

We perform this optimization regularly when the cell-load, channel or user position changes. For sum-rate and throughput computation, we can have closed-form expressions with respect to given channel link gains.

\section{Performance evaluation}

We validate the proposed joint processing scheme in two steps. First, we check whether symbols can be accurately detected for the jointly scheduled users. Secondly, we evaluate the throughput improvement in the case where many users are in each cell and jointly detected users are selected between them.

In our first simulation study, we implement a single carrier model where one user in each cell transmits simultaneously towards the receivers (RRHs) who share the received signals for joint detection using multi-cell MMSE. We use LTE urban macro-cell channel model [24], where 16-QAM modulated symbols are sent. We compare error rate after QAM demodulation without any repeat mechanism. Since our joint detection scheme does not affect user-PHY processing, it is appropriate to evaluate error rate at this point. Fig. 3 shows the error rates in the following cases for comparison: (i) interference-free transmission, (ii) 2-user cooperation, (iii) 3-user cooperation, and (iv) non-cooperative detection.

We see that in the JD cases we need slightly higher transmit power to reach the same error rate as in singleuser transmission, but we transmit on one PRB only instead of using one for each user. This confirms that if frequency resources become scarce, using JD in the BBU-pool enables accurate high-rate transmission on the uplink. We notice also that higher cluster-size requires higher power since meansquare error at detection can increase with the dimension of received signal. It appears also clearly that without cooperation

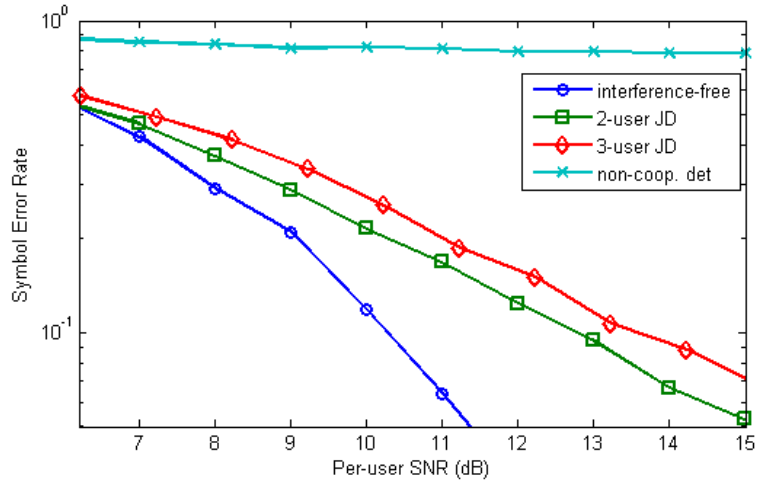

Fig. 3: A comparison of the symbol error rates: interferencefree transmission, 2-user joint detection, 3-user joint detection, and non-cooperative detection.

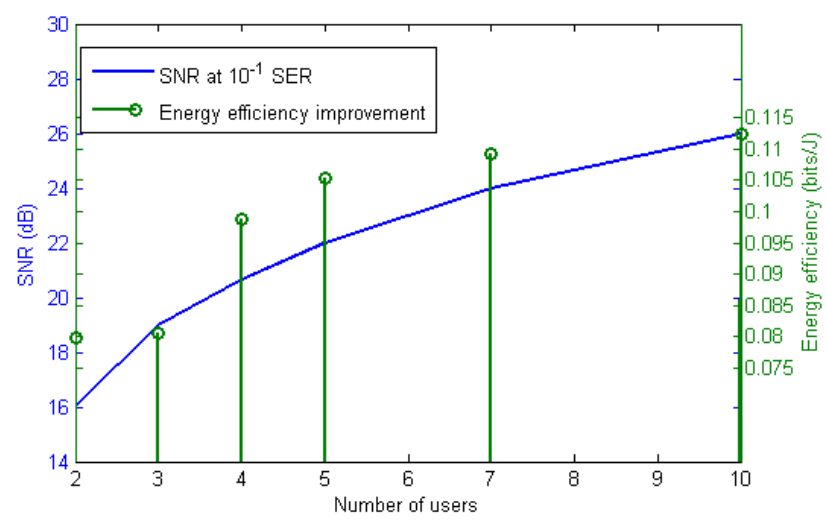

Fig. 4: Required SNR for achieving $10^{-1}$ symbol error rate during the joint detection and improvement of energy efficiency w.r.t. the 1 user/PRB case.

at the receiver side, the SINR is too low to perform accurate detection.

We show in Fig. 4 the overall SNR $=\frac{\sum_{i} \sum_{j}\left|h_{i j} x_{i}\right|^{2}}{\sigma_{n}^{2}}$ required for achieving symbol error rate of $10^{-1}$ in joint detection for different cooperation cluster sizes and the improvement of energy efficiency using JD with respect to the case when each user transmits on a different PRB. The number of cooperating RRHs is set equal to the number of interfering users in each joint detection. We observe that per-user transmission power needs to be increased with the size of the cooperation cluster, but the required additional power for adding a user becomes lower as the cluster size increases. Thus, energy efficiency gain increases with cluster size. Note that in general the symbol error rate can be improved by some retransmission and coding schemes for low bit error rate criteria.

As the complexity of the joint MMSE detection scales as $O\left(n^{2}\right)$ where $n$ is the number of jointly detected users, we studied the possibility of reducing this complexity by realizing the JD over a subset of the cooperation cluster only. This would allow jointly detected users to benefit from CSI of all 
co-channel users, but we should deal with interference coming from non-JD users. In addition, it turns out that for users transmitting with the same power and having pathlosses of the same order, overall SINR $=\frac{\sum_{i \neq k} \sum_{j}\left|h_{i j} x_{i}\right|^{2}}{\sum_{j}\left|h_{k j} x_{k}\right|^{2}+\sigma_{n}^{2}}$ does not scale with the transmission power, so JD cannot achieve target error rate unless all co-channel users are jointly detected. Note that to handle potential implementation complexity, one can also consider to distribute the joint MMSE function among BBUs, in a parallelized system implementation.

To evaluate how much the system average throughput and cell-edge throughput are improved by user selective joint detection, in our current work we are implementing system simulations with realistic traffics. By adding a joint scheduling optimization algorithm and joint detection function for selected subcarriers in full-stack LTE base stations, we can simulate a full-load network scenario where we expect system performance improvement.

\section{CONCLUSION AND Future Work}

In this paper, we explore C-RAN architecture as an enabler for CoMP, including its limitations and the perspectives for next-generation cellular networks. We investigate the network elements required for C-RAN such as SDN-controller and evolved fronthaul transport. They can greatly facilitate the implementation of uplink cooperation between users, taking into account the practical limitations. The proposed joint detection method satisfies real network constraints and can improve cell throughput and cell-edge QoS.

In the next step of our work, we plan to transfer this implementation to real-time software C-RAN platform, where we can evaluate the benefit of the inter-cell coordination and joint processing as a northbound application with the SDN controller in our testbed. We can also evaluate the impact of adding this joint detection and its actual computation time. The evolution of C-RAN from today's physical BBU centralization towards future SDN-based dynamic configurations is in line with our idea of having a functional split in the physical layer with the possibility of placing user-PHY processing either in the BBU-pool or in the RRH. Benefiting from the possibility of low-latency multi-cell cooperation in C-RAN, it is expected that the proposed approach and user selective uplink joint detection scheme would be important for addressing inter-cell interference problems in future very dense cellular networks.

\section{ACKNOWLEDGEMENT}

Special thanks to J. Hoydis for precious comments and Y. El Mghazali for discussions and ideas about future networks. This work was supported in part by the 7th Framework Programme for Research of the European Commission HARP project under Grant HARP-318489.

\section{REFERENCES}

[1] J. G. Andrews, S. Buzzi, W. Choi, S. V. Hanly, A. E. Lozano, A. C. K Soong, and J. C. Zhang, "What will 5G be?" IEEE Journal on Selected Areas in Communications, vol. 32, no. 6, pp. 1065-1082, 2014.
[2] D. Gesbert, S. Hanly, H. Huang, S. S. Shitz, O. Simeone, and W. Yu, "Multi-cell MIMO cooperative networks: A new look at interference," IEEE J. Sel. A. Commun., vol. 28, no. 9, pp. 1380-1408, Dec. 2010.

[3] C. Singh and C. S. Chen, "Distributed downlink resource allocation in cellular networks through spatial adaptive play," in 25th International Teletraffic Congress (ITC), Sept 2013, pp. 1-9.

[4] P. Marsch and G. Fettweis, "Uplink CoMP under a constrained backhaul and imperfect channel knowledge," IEEE Transactions on Wireless Communications, vol. 10, no. 6, pp. 1730-1742, 2011.

[5] S. V. Hanly, "Capacity and power control in spread spectrum macrodiversity radio networks," IEEE Transactions on Communications, vol. 44 no. 2, pp. 247-256, 1996

[6] P. Marsch and G. P. Fettweis, Coordinated Multi-Point in Mobile Communications: From Theory to Practice. Cambridge University Press, 2011.

[7] L. Zhou and W. Yu, "Uplink multicell processing with limited backhaul via per-base-station successive interference cancellation," IEEE J. Sel. A. Commun., vol. 31, no. 10, pp. 1981-1993, Oct. 2013.

[8] K. Miyamoto, S. Kuwano, J. Terada, and A. Otaka, "Uplink joint reception with LLR forwarding for optical transmission bandwidth reduction in mobile fronthaul," in IEEE VTC-Spring, 2015, pp. 1-5.

[9] A. Sanderovich, O. Somekh, H. Poor, and S. Shamai, "Uplink macro diversity of limited backhaul cellular network," IEEE Transactions on Information Theory, vol. 55, no. 8, pp. 3457-3478, Aug 2009.

[10] K. Balachandran, J. Kang, K. Karakayali, and K. REge, "Nice: A network interference cancellation engine for opportunistic uplink cooperation in wireless networks," IEEE Transactions on Wireless Communications, vol. 10, no. 2, pp. 540-549, Feb. 2011.

[11] 3GPP, "Evolved Universal Terrestrial Radio Access (E-UTRA); Physical Channels and Modulation," 3rd Generation Partnership Project (3GPP), TR 36.211, 2014.

[12] — "X2 application protocol (X2AP)," 3rd Generation Partnership Project (3GPP), TR 36.423, 2010.

[13] A. Checko, H. Christiansen, Y. Yan, L. Scolari, G. Kardaras, M. Berger, and L. Dittmann, "Cloud RAN for mobile networks - a technology overview," IEEE Communications Surveys and Tutorials, vol. 17, no. 1, pp. 405-426, 2014.

[14] D. Sabella, P. Rost, Y. Sheng, E. Pateromichelakis, U. Salim, P. GuittonOuhamou, M. Di Girolamo, and G. Giuliani, "RAN as a service: Challenges of designing a flexible RAN architecture in a cloud-based heterogeneous mobile network," in Future Network and Mobile Summit, July 2013, pp. 1-8.

[15] C.-L. I, Y. Yuan, J. Huang, S. Ma, C. Cui, and R. Duan, "Rethink fronthaul for soft RAN," IEEE Communications Magazine, vol. 53, no. 9, pp. 82-88, September 2015.

[16] S. Yeganeh, A. Tootoonchian, and Y. Ganjali, "On scalability of software-defined networking," IEEE Communications Magazine, vol. 51, no. 2, pp. 136-141, Feb. 2013

[17] M. Mendonca, K. Obraczka, and T. Turletti, "The case for softwaredefined networking in heterogeneous networked environments," in ACM Conference on CoNEXT Student Workshop, 2012, pp. 59-60.

[18] A. R. Curtis, J. C. Mogul, J. Tourrilhes, P. Yalagandula, P. Sharma, and S. Banerjee, "DevoFlow: Scaling flow management for highperformance networks," SIGCOMM Comput. Commun. Rev., vol. 41, no. 4, pp. 254-265, Aug. 2011.

[19] K.-K. Yap, M. Kobayashi, R. Sherwood, T.-Y. Huang, M. Chan, N. Handigol, and N. McKeown, "OpenRoads: Empowering research in mobile networks," SIGCOMM Comput. Commun. Rev., vol. 40, no. 1, pp. 125-126, Jan. 2010.

[20] M. Bansal, J. Mehlman, S. Katti, and P. Levis, "OpenRadio: A programmable wireless dataplane," in First Workshop on Hot Topics in Software Defined Networks, 2012, pp. 109-114.

[21] A. Gopalasingham, L. Roullet, N. Trabelsi, C. S. Chen, A. Hebbar, and E. Bizouarn, "Generalized software defined network platform for radio access networks," in IEEE Consumer Communications and Networking Conference, 2016

[22] C. M. R. Institute, "C-RAN - the road towards green RAN," 2011.

[23] S. Sesia, I. Toufik, and M. Baker, LTE - The UMTS Long Term Evolution: From Theory to Practice. Wiley Publishing, 2009.

[24] 3GPP, "Spatial channel model for Multiple Input Multiple Output (MIMO) simulations ," 3rd Generation Partnership Project (3GPP), TR 25.996, 2014. 